\title{
Criação e validação de aplicativo para avaliação de dor infantil
}

\author{
Creation and validation of an application for child pain assessment \\ Creación y validación de una aplicación para la evaluación del dolor infantil
}

Recebido: 29/06/2021 | Revisado: 14/07/2021 | Aceito: 16/08/2021 | Publicado: 17/08/2021

\author{
Valmor Arêde Córdova Júnior \\ ORCID: https://orcid.org/0000-0002-6926-5774 \\ Fundação Santa Casa de Misericórdia do Pará, Brasil \\ E-mail: valmor_cordova@yahoo.com.br \\ Silvestre Savino Neto \\ ORCID: https://orcid.org/0000-0002-2350-1022 \\ Fundação Santa Casa de Misericórdia do Pará, Brasil \\ E-mail: savino@ufpa.br \\ David Santos Cabral \\ ORCID: https://orcid.org/0000-0001-5662-5008 \\ Centro Universitário do Pará, Brasil \\ E-mail: davidcabral252001@gmail.com \\ Aurimery Gomes Chermont \\ ORCID: https://orcid.org/0000-0001-8715-3576 \\ Fundação Santa Casa de Misericórdia do Pará, Brasil \\ E-mail: agchermont@gmail.com \\ Creusa Barbosa dos Santos Trindade \\ ORCID: https://orcid.org/0000-0001-7482-1475 \\ Fundação Santa Casa de Misericórdia do Pará, Brasil \\ E-mail: cbcreusasantos7@gmail.com
}

\begin{abstract}
Resumo
O manejo da dor infantil é complexo devido as limitações de comunicação das crianças e tem seu ponto de partida em uma avaliação adequada da dor, contudo há uma gama de escalas de avaliação da dor e os profissionais em dificuldade em escolher a ferramenta adequada. Objetivo: construir e validar aplicativo de avaliação de dor em crianças a partir do estudo das escalas disponíveis. Metodologia: Trata-se de um estudo descritivo de desenvolvimento experimental. A pesquisa se desenvolveu nas seguintes etapas: desenvolvimento do aplicativo, validação por especialistas e análise de dados. Realizou-se a revisão exploratória sobre as escalas de avaliação de dor em crianças para fundamentar o aplicativo. $\mathrm{O}$ desenvolvimento do aplicativo foi realizado em parceria com profissional de computação a fim de fornecer uma interface amigável. A validação foi realizada por especialistas profissionais de saúde (médicos, enfermeiros e fisioterapeutas) através de um questionário pré-estabelecido contendo informações sobre a formação acadêmica e profissional dos validadores, e sobre os itens a serem validados. Resultados: Para análise de dados, utilizou-se o índice de validade de conteúdo (IVC), sendo considerado válido quando o quesito obtivesse IVC > 0.8. O aplicativo foi validado com IVC de 1.0 pelos especialistas. Conclusão: A avaliação positiva do aplicativo demonstra que é uma ferramenta válida e com boa aceitação pelos profissionais de saúde envolvidos nos cuidados da dor em crianças e que a incorporação de novas tecnologias pode otimizar e facilitar o uso de escalas na avaliação de dor em crianças.
\end{abstract}

Palavras-chave: Medição da dor; Crianças; Saúde da criança; Aplicativos móveis; Tecnologia biomédica.

\begin{abstract}
Child pain management is complex due to children's communication limitations and has its starting point in an adequate pain assessment, however there is a range of pain assessment scales and professionals in difficulty in choosing the appropriate tool. Objective: build and validate an application for pain assessment in children based on the study of the available scales. Methodology: This is a descriptive study of experimental development. The research was carried out in the following stages: application development, validation by experts and data analysis. An exploratory review was carried out on the scales of pain assessment in children to support the application. The development of the application was carried out in partnership with a computing professional in order to provide a friendly interface. Professional health specialists (doctors, nurses and physiotherapists) through a pre-established questionnaire containing information about the academic and professional training of the validators, and about the items to be validated carried out the validation. Results: For data analysis, the content validity index (CVI) was used, being considered valid when the item obtained CVI $>0.8$. The experts have validated the application with a CVI of 1.0. Conclusion: The positive evaluation of the application demonstrates that it is a valid tool and with good acceptance by health professionals involved in the care of pain in children and that, the incorporation of new technologies can optimize and facilitate the use of scales in the evaluation of pain in children.
\end{abstract}

Keywords: Pain measurement; Kids; Child health; Mobile apps; Biomedical technology. 


\begin{abstract}
Resumen
El manejo del dolor infantil es complejo debido a las limitaciones de comunicación de los niños y tiene su punto de partida en una adecuada evaluación del dolor, sin embargo existe una gama de escalas de evaluación del dolor y profesionales con dificultad para elegir la herramienta adecuada. Objetivo: construir y validar una aplicación para la evaluación del dolor en niños basada en el estudio de las escalas disponibles. Metodología: Este es un estudio descriptivo del desarrollo experimental. La investigación se llevó a cabo en las siguientes etapas: desarrollo de la aplicación, validación por expertos y análisis de datos. Se realizó una revisión exploratoria de las escalas de evaluación del dolor en niños para apoyar la aplicación. El desarrollo de la aplicación se llevó a cabo en colaboración con un profesional de la informática para proporcionar una interfaz amigable. La validación fue realizada por profesionales especialistas en salud (médicos, enfermeras y fisioterapeutas) a través de un cuestionario preestablecido que contenía información sobre la formación académica y profesional de los validadores, y sobre los ítems a validar. Resultados: Para el análisis de los datos se utilizó el índice de validez de contenido (IVC), considerándose válido cuando el ítem obtuvo un IVC> 0,8. La aplicación ha sido validada con un CVI de 1.0 por los expertos. Conclusión: La valoración positiva de la aplicación demuestra que se trata de una herramienta válida con buena aceptación por parte de los profesionales sanitarios implicados en el cuidado del dolor en niños y que la incorporación de nuevas tecnologías puede optimizar y facilitar el uso de escalas en la valoración del dolor en niños.

Palabras clave: Medición del dolor; Niños; Salud de los niños; Aplicaciones móviles; Tecnología biomédica.
\end{abstract}

\title{
1. Introdução
}

A dor é uma experiência sensorial e emocional desagradável, multifatorial, subjetiva e deve ser avaliada, acompanhada e tratada respeitando seus aspectos subjetivos. O manejo da dor na criança é mais complexo que no adulto, pois pode haver limitações na avaliação decorrente das dificuldades de percepção e comunicação das crianças, as quais estão em desenvolvimento. O impacto da dor na criança supera o aspecto físico e psicossocial, também acarreta limitações funcionais, prejudica a atividade física, o apetite e o sono, podendo debilitar ainda mais o paciente. Os profissionais de saúde devem ser capazes de avaliar o quadro álgico e promover o alívio do sofrimento doloroso. Para tal, há diversas ferramentas e técnicas de avaliação da dor infantil e vasto arsenal terapêutico (Marec-Berard, et al., 2015).

O manejo adequado da dor em crianças é complexo e exige uma abordagem multiprofissional, a qual se inicia e tem como pilar básico a avaliação apropriada e de forma sistemática e cientificamente válida da dor. As escalas de avaliação da dor permitem uma abordagem adequada da dor, contudo há uma gama significativa de escalas à disposição dos profissionais de saúde. Apesar da reconhecida importância da avaliação e mensuração da dor no manejo adequado do quadro álgico, os profissionais de saúde, os quais estão diretamente ligados ao cuidado da criança, relatam dificuldade em avaliar a intensidade da dor e apresentam pouco conhecimento sobre instrumentos de avaliação de dor (Salum, et al., 2012).

É importante escolher o melhor instrumento de acordo com a capacidade cognitiva e de interação da criança para que se obtenham informações fidedignas do quadro doloroso, o uso inadequado destes instrumentos na avaliação da dor pode comprometer a prática clínica. Escolher a escala que melhor se adapte ao paciente é uma tarefa árdua, pois na avaliação da dor deve-se levar em conta não somente dados mensuráveis e objetivos, mas também aspectos biopsicossociais da criança, portanto pode ser interessante o uso de mais de uma ferramenta na avaliação da dor (Santos, et al, 2017).

Apesar da dor ser uma fonte significativa de preocupação das crianças e seus familiares, nota-se que a avaliação da dor não é realizada sistematicamente pela equipe multiprofissional. Tempo limitado, grande quantidade de documentos a ser preenchidos no plantão, desconhecimento das escalas são algumas barreiras apontadas para a não utilização rotineira das escalas de avaliação de dor (Fortier, et al., 2016).

A avaliação dinâmica em tempo real por meio de tecnologia móvel é uma possibilidade viável para facilitar a avaliação da dor. A comunicação eletrônica tem sido bem recebida no âmbito da saúde pelo público infanto-juvenil, os meios eletrônicos de informação aumentam a aderência ao monitoramento dos dados de informações em saúde e promovem maior engajamento nos cuidados em saúde. Aplicativos eletrônicos de avaliação da dor já se mostraram úteis na rotina hospitalar, 
uma vez que foram capazes de detectar mudanças no complexo padrão diário de dor e fornecer informações fidedignas em tempo real para o tratamento da dor (Stinson, et al., 2015).

O desenvolvimento de soluções computacionais em forma de aplicativo móvel tem se tornado um meio eficaz de disponibilizar ferramentas resolutivas para um determinado público alvo de modo acessível e rápido. As tecnologias em saúde estão em célere expansão, pois proporcionam maior agilidade e precisão no apoio diagnóstico, tomada de decisão, avaliação e tratamento. Tendo em vista as dificuldades e limitações pertinente ao processo de avaliação da dor infantil, este estudo objetivou criar e validar um aplicativo de smartphone para avaliação da dor em crianças a partir das escalas de avaliação disponíveis e validadas para uso em crianças (Sarno, et al., 2014).

\section{Metodologia}

Realizou-se um estudo descritivo de desenvolvimento experimental para construção de um aplicativo de dispositivo móvel, com abordagem quantitativa. Os estudos de desenvolvimento experimental são realizados a partir de conhecimentos preexistentes e buscam demonstrar a viabilidade técnica ou funcional, seja de novos produtos, processos, sistemas ou serviços. O desenvolvimento experimental permite a inovação tecnológica e a criação de produtos a partir de conhecimentos científicos pregressos para aplicação no dia a dia (Ipdmaq, 2016).

O estudo foi desenvolvido em parceria com um profissional de ciências da computação, com reuniões virtuais para criação e desenvolvimento do aplicativo a partir das escalas de avaliação da dor selecionadas e validadas para uso com crianças e frequentemente utilizadas em pesquisas científicas e na prática clínica.

O aplicativo foi desenvolvido para auto avaliação da dor em crianças, com o intuito de que disponha as escalas de avaliação de modo prático e objetivo. Propõe-se a ser utilizado em pediatria com crianças a partir de 08 anos até 18 anos, uma vez que crianças a partir de 8 anos são capazes de fornecer dados confiáveis através de aplicativos para smartphones (Tutelman, et al., 2018).

O aplicativo engloba escalas de auto avaliação de dor já validadas de modo sistemático e prático para que se permita ser viável e fidedigno avaliar o quadro doloroso das crianças. Foram usadas como base para o desenvolvimento do aplicativo as seguintes escalas: EFWB, EVA, EVN, mapa de diagrama corporal.

$\mathrm{O}$ aplicativo foi construído com a ferramenta de prototipação Adobe XD, sendo baseado em fundamentos de UI/UX para oferecer a melhor interface para os usuários. No âmbito da programação, foi utilizado o React Native, framework criado em 2015 pelo Facebook, o qual usa a linguagem JavaScript para a criação de aplicativos mobile de forma nativa, levando a fornecer uma melhor construção da interface para o usuário, tendo sua primeira versão apenas para sistemas operacionais Android.

Após o término do desenvolvimento do protótipo do aplicativo, este foi submetido a validação de especialistas, mediante avaliação técnica através de questionário semiestruturado criado pelo pesquisador e aplicado à profissionais de saúde envolvidos diretamente nos cuidados da dor infantil. Considerou-se médicos, enfermeiros e fisioterapeutas como corpo técnico de profissionais para validação.

O questionário foi entregue aos especialistas no momento da avaliação do aplicativo. Utilizou-se no questionário escala tipo Likert a qual determina a concordância favorável ou desfavorável dos entrevistados diante das assertivas apresentadas. O questionário foi dividido em duas partes, sendo o primeiro bloco compostos por questões demográficas como idade, sexo, profissão, grau e tempo de formação acadêmica e tempo de atuação em pediatria. O segundo bloco era composto da avaliação do aplicativo, contendo oito perguntas em que se avaliou quesitos de funcionalidade, usabilidade e eficiência, os quais abrangem os aspectos qualitativos fundamentais para o usuário do software (Tibes, 2014). 
Foram incluídos no estudo médicos, fisioterapeutas e enfermeiros com titulação mínima de especialização lato sensu e com experiência profissional mínima de dois anos na área de pediatria. Foram excluídos da pesquisa os profissionais que relataram não possuir familiarização com uso de aplicativos de smartphone, aqueles que desmarcaram mais de duas vezes a avaliação, os que se recusaram a participar da pesquisa e que não estejam em atuação profissional na pediatria no momento da validação da pesquisa (Tibes, 2014).

Para que a amostra de avaliação de produtos de engenharia de software seja significativa em relação ao número de usuários, a validação deve ser conduzida com no mínimo oito avaliadores de cada categoria de usuário. A amostra dos validadores ocorreu por conveniência e fez parte da amostra profissionais da Fundação Santa Casa de Misericórdia do Pará e do Hospital Universitário João de Barros Barreto, ambos hospitais terciários localizado na cidade de Belém-PA.

O uso do protótipo experimental do aplicativo pelo especialista e aplicação do questionário foi realizado individualmente com cada avaliador nos meses de março e abril de 2021. O uso do aplicativo e resposta ao questionário foi realizado pelo avaliador no seu local de trabalho. Todos os participantes receberam, no momento da validação, um aparelho celular com o aplicativo já instalado e configurado, facilitando o manuseio. Considerou-se validadas as respostas marcadas com classificação "concordo totalmente" e "concordo parcialmente".

Para a validação de conteúdo, utilizou-se o índice de validade de conteúdo (IVC). Adotou-se valor de concordância > 0,8 entre avaliadores para validação do conteúdo do aplicativo. O IVC mede a proporção ou porcentagem de especialistas que estão em concordância sobre determinados aspectos do instrumento e de seus itens. O cálculo do IVC é feito através da média do número de respostas válidas. Para análise estatística de dados, as respostas foram previamente tabuladas em uma planilha do Excel. A análise estatística foi do tipo descritiva (Pasquali, 2010).

\section{Resultados}

O aplicativo se divide em 04 módulos apresentados em sequência cronológica. São estes: tela inicial, módulo introdutório ou de apresentação, módulo sociodemográfico, módulo de escalas de avaliação e módulo de relatório da avaliação da dor.

A tela inicial apresenta uma mensagem de boas-vindas na parte superior da tela em destaque numa caixa azul, na porção central está a mensagem "Vamos começar sua avaliação" juntamente com o ícone e nome do aplicativo, e na região inferior há o ícone "começar" (Figura 1).

A tela seguinte comporta o módulo de introdução do aplicativo, nesta etapa é explicado sucintamente que serão apresentadas à criança as escalas de avaliação para verificar o nível de sua dor naquele momento, em seguida a esta mensagem introdutória são descritas as escalas que compõem o aplicativo. Posteriormente, há orientação para que a criança responda adequadamente o que será solicitado na avaliação (Figura 2). 
Figura 1. Tela Inicial.

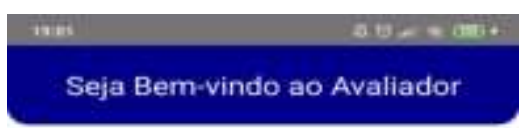

Vamos Começar a sua Avaliaçāoll!

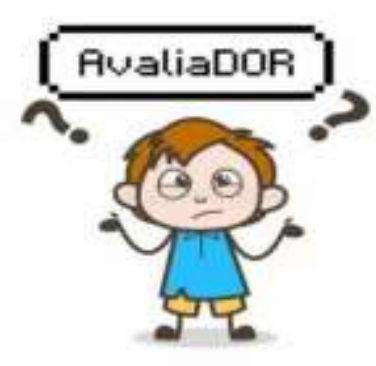

Começar

Fonte: Autores (2021).

Figura 2. Tela de introdução.
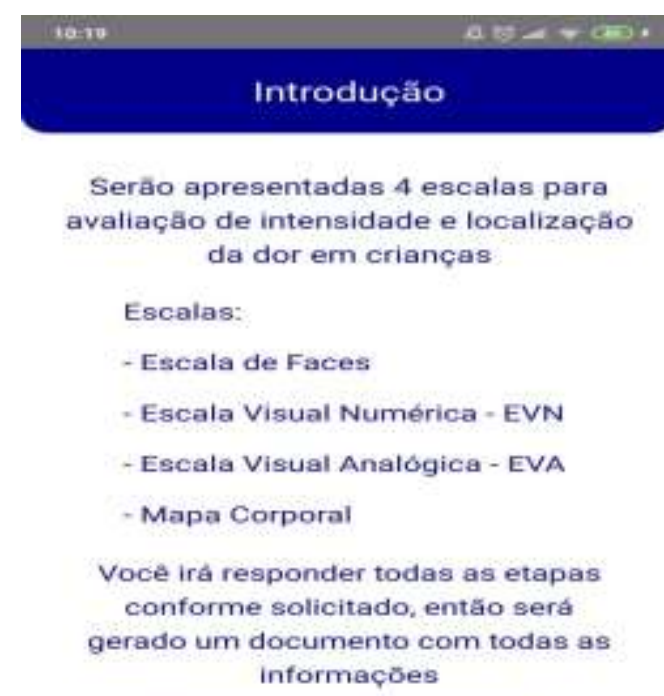

Seräo apresentadas 4 escalas para avaliaça de intensidade e localizaçăo da dor em crianças

Escalas:

- Escala de Faces

- Escala Visual Numerica - EVN

- Escala Visual Analogica - EVA

- Mapa Corporal

Voce ira responder todas as etapas conforme solicitado, então será gerado um documento com todas as informaçöes

\section{Começar Avaliaçäo}

Fonte: Autores (2021).

O terceiro módulo refere-se aos dados sociodemográficos, onde são cadastradas as seguintes informações pessoais do paciente: nome, idade e local de avaliação. O quarto módulo é composto cronologicamente pelas seguintes escalas de avaliação: EFWB, EVA, EVN e Mapa Corporal (figuras 3,4,5,6, respectivamente). Nestas etapas de avaliação, o aplicativo foi programado para rotacionar para visualização horizontal, a fim de facilitar o processo de avaliação. Na porção superior das telas de avaliação, há o nome de cada escala e logo abaixo um breve texto de orientação para nortear a avaliação. Na região central da tela, temos as escalas em si a serem preenchidas. 
Figura 3. Tela aplicativo da Escala de Faces de Wong Baker.

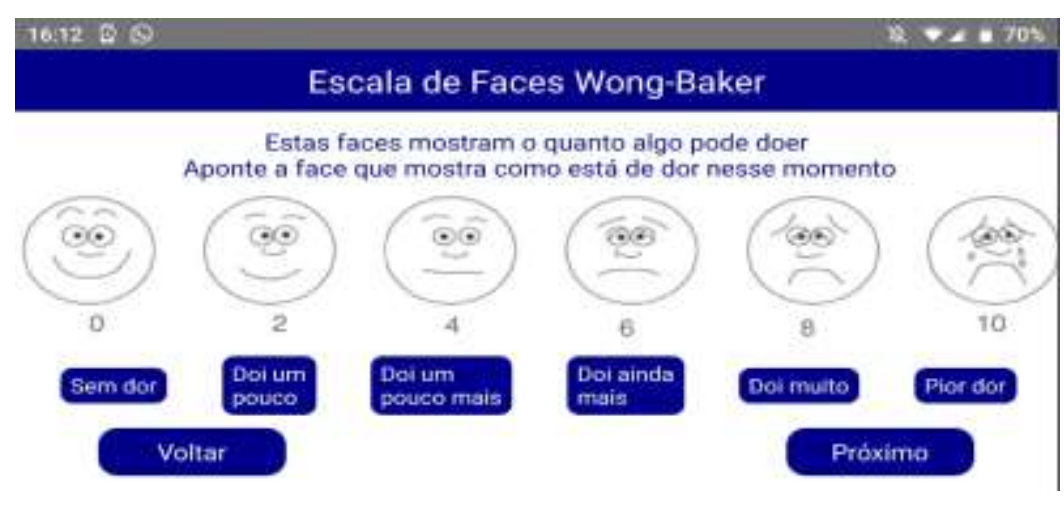

Fonte: Autores (2021).

Figura 4. Tela aplicativo da Escala Visual Numérica.

Escala Visual Numérica

Sendo 0 sem dor e 10 a pior dor possivel, como está sua dor nesse momento?

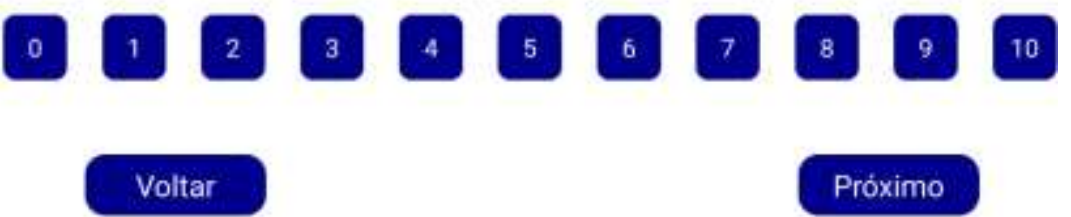

Fonte: Autores (2021).

Figura 5. Tela aplicativo da Escala Visual analógica.

Escala Visual Analógica

De sem dor a pior dor possivel, como está sua dor nesse momento?

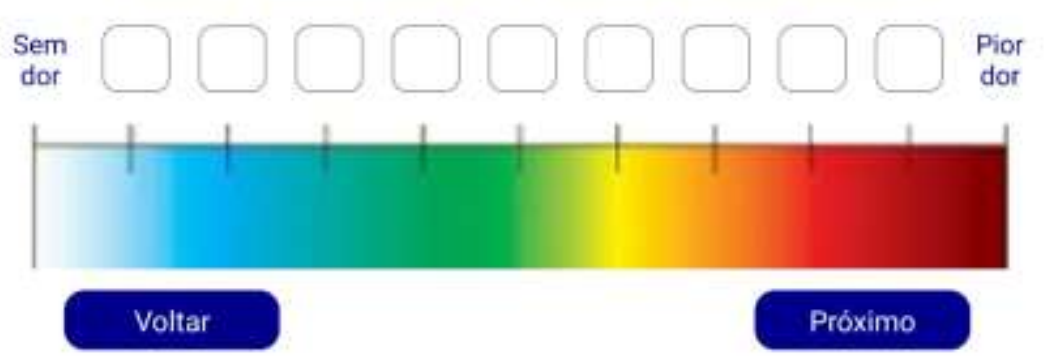

Fonte: Autores (2021). 
Figura 6. Tela aplicativo da Escala de Mapa Corporal.

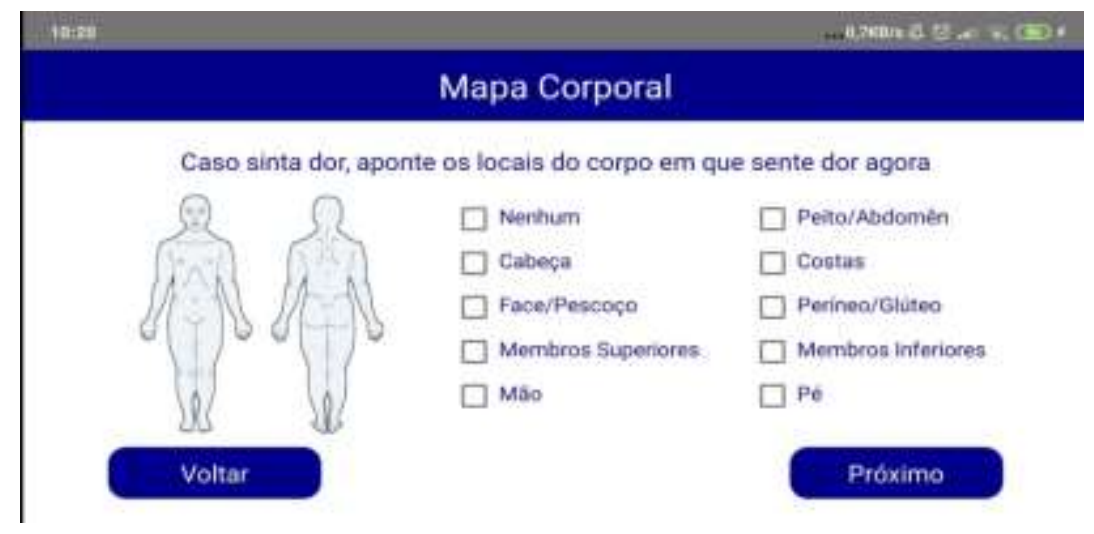

Fonte: Autores (2021).

A última etapa do aplicativo é o módulo de formulário da dor, que consiste em um relatório resumido com os dados do paciente de nome, idade, local e data da avaliação e as informações dos resultados da intensidade e localização da dor de acordo com cada escala dispostos de maneira prática e intuitiva, este formulário é salvo na pasta de imagens do celular e pode ser consultado a posteriori pelo profissional. (Figura 7).

Figura 7. Formulário de relatório final da dor.

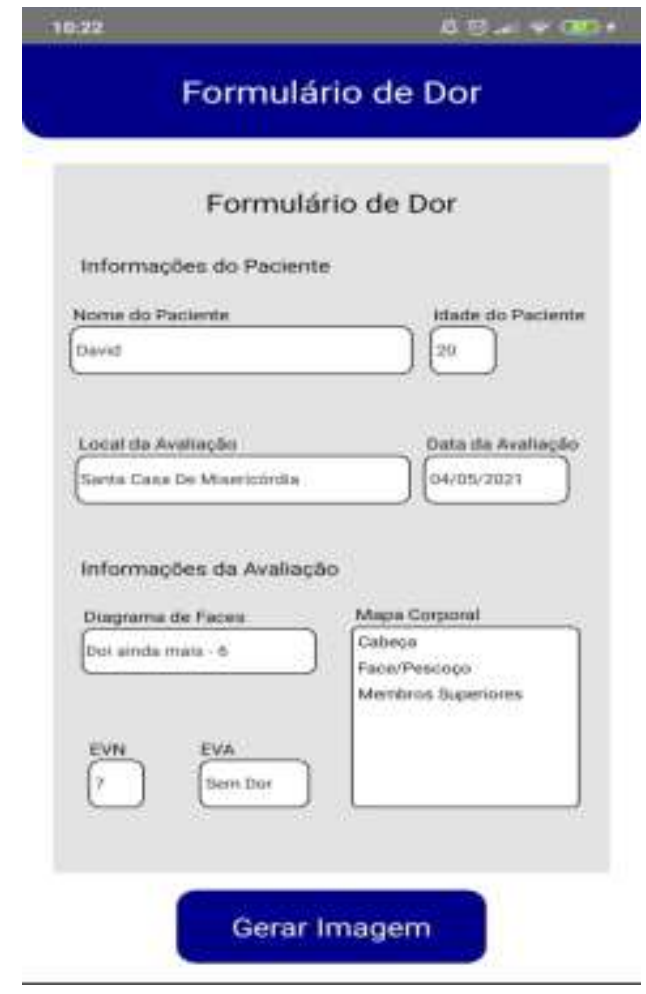

Fonte: Autores (2021).

Participaram da pesquisa um total de 12 profissionais de saúde avaliadores especialistas, sendo 08 (oito) do sexo feminino e 04 (quatro) do sexo masculino. No que se refere a idade, apenas 01 (uma) tinha menos do que 30 anos, 08 (oito) possuíam entre 30 e 39 anos e 03 (três) apresentavam mais de 40 anos.

Destes profissionais, 04 (quatro) eram médicos, 04 (quatro) enfermeiros e 04 (quatro) fisioterapeutas. Quanto ao grau de titulação acadêmica, 07 (sete) eram especialistas e 05 (cinco) eram mestres (Tabela 1). Em relação ao tempo de graduação, 
08 (oito) graduaram-se há mais de 10 anos e 04 (quatro) haviam se graduados entre 05 a 10 anos. Sobre o tempo de experiência na área de pediatria, 03 (três) possuíam entre 02 a 05 anos, 04 (quatro) entre 05 a 10 anos e 05 (cinco) profissionais trabalhavam há mais de 10 anos em pediatria (Tabela 2).

Tabela 1. Profissão e titulação acadêmica dos avaliadores.

\begin{tabular}{cl}
\hline Variável & N \\
\hline Formação & 04 \\
Médico & 04 \\
Enfermeiro & 04 \\
Fisioterapeuta & $\mathbf{1 2}$ \\
Total & \\
& \\
Titulação acadêmica & \\
Residência/Especialista & 07 \\
Mestrado & 05 \\
Total & $\mathbf{1 2}$ \\
\hline
\end{tabular}

Fonte: Autores (2021).

Tabela 2. Tempo de graduação e experiência pediátrica.

\begin{tabular}{cl}
\hline Variável & $\mathbf{N}$ \\
\hline Tempo de graduação & \\
05 a 10 anos & 04 \\
Mais que 10 anos & 08 \\
Total & $\mathbf{1 2}$ \\
& \\
Tempo de experiência em pediatria & \\
02 a 05 anos & 03 \\
05 a 10 anos & 04 \\
Mais de 10 anos & 05 \\
Total & $\mathbf{1 2}$ \\
\hline
\end{tabular}

Fonte: Autores (2021).

No que se refere a validação do aplicativo, os avaliadores responderam a questões referentes a funcionalidade (03 perguntas), usabilidade (03 perguntas) e eficiência (02 perguntas). Quanto a funcionalidade, dois juízes concordaram parcialmente sobre a disposição das funções necessárias para avaliação da dor infantil e precisão na execução das funções, sendo que os demais concordaram totalmente. Sobre a facilidade do uso do aplicativo, os juízes foram unânimes em concordarem totalmente (Tabela 3) 
Tabela 3. Respostas questões de funcionalidade.

\begin{tabular}{|c|c|c|c|c|c|c|}
\hline $\begin{array}{c}\text { Questões de } \\
\text { Funcionalidade }\end{array}$ & $\begin{array}{c}\text { Discordo } \\
\text { total }\end{array}$ & $\begin{array}{c}\text { Discordo } \\
\text { parcial }\end{array}$ & $\begin{array}{l}\text { Nem concordo, nem } \\
\text { discordo }\end{array}$ & $\begin{array}{c}\text { Concordo } \\
\text { parcial }\end{array}$ & Concordo total & IVC \\
\hline $\begin{array}{l}\text { Dispõe das funções para } \\
\text { avaliar e classificar a dor }\end{array}$ & - & - & - & 02 & 10 & 1.0 \\
\hline $\begin{array}{c}\text { Precisão na execução das } \\
\text { funções }\end{array}$ & - & - & - & 02 & 10 & 1.0 \\
\hline $\begin{array}{l}\text { Facilidade no uso do } \\
\text { aplicativo }\end{array}$ & - & - & - & - & 12 & 1.0 \\
\hline
\end{tabular}

Fonte: Autores (2021).

Em relação à usabilidade, um avaliador concordou parcialmente sobre o entendimento do conceito do aplicativo e o restante concordou totalmente. Três avaliadores relataram concordância parcial quanto a ajuda do aplicativo e nove relataram concordância total. Os doze avaliadores apresentaram consenso quanto a facilidade de aprendizado no uso do aplicativo (Tabela 4).

Tabela 4. Respostas questões de usabilidade.

\begin{tabular}{|l|c|c|c|c|c|c|}
\hline Questões de Usabilidade & $\begin{array}{l}\text { Discordo } \\
\text { total }\end{array}$ & $\begin{array}{l}\text { Discordo } \\
\text { parcial }\end{array}$ & $\begin{array}{l}\text { Nem concordo, nem } \\
\text { discordo }\end{array}$ & $\begin{array}{l}\text { Concordo } \\
\text { parcial }\end{array}$ & $\begin{array}{l}\text { Concordo total } \\
\text { IVC }\end{array}$ & 1.0 \\
\hline $\begin{array}{l}\text { Entender o conceito do } \\
\text { aplicativo }\end{array}$ & - & - & - & - & 11 \\
\hline $\begin{array}{l}\text { Aprendizado do uso do } \\
\text { aplicativo }\end{array}$ & - & - & - & 01 & 1.0 \\
\hline Ajuda de forma clara & - & - & - & 03 & 1.0 \\
\hline
\end{tabular}

Fonte: Autores (2021).

No que tange a eficiência do aplicativo, todos os avaliadores concordaram totalmente que o aplicativo tem tempo de execução adequado. Sobre o quesito a respeito dos recursos do aplicativo, cinco concordaram parcialmente e sete concordaram totalmente (Tabela 5).

Tabela 5. Questões de eficiência.

\begin{tabular}{|l|l|l|l|c|c|}
\hline Questões de Eficiência & $\begin{array}{l}\text { Discordo } \\
\text { total }\end{array}$ & $\begin{array}{l}\text { Discordo } \\
\text { parcial }\end{array}$ & $\begin{array}{l}\text { Nem concordo, nem } \\
\text { discordo }\end{array}$ & $\begin{array}{l}\text { Concordo } \\
\text { parcial }\end{array}$ & Concordo total \\
IVC \\
\hline $\begin{array}{l}\text { Tempo de execução } \\
\text { adequado }\end{array}$ & - & - & - & - & 12 \\
\hline $\begin{array}{l}\text { Recursos adequados do } \\
\text { aplicativo }\end{array}$ & - & - & - & 05 & 07 \\
\hline
\end{tabular}

Fonte: Autores (2021).

Em relação ao IVC, todos os itens foram validados pelos juízes, tendo como respostas "concordo parcialmente" e “concordo totalmente" para os questionamentos apresentando e obtendo IVC de 1.0 para totalidade dos itens avaliados. 


\section{Discussão}

A tecnologia móvel pode ser aplicada em diversas vertentes da saúde. Dentre essas, destaca-se o monitoramento remoto, o apoio diagnóstico, a tomada de decisão e segurança do paciente. Isto corrobora o uso do aplicativo, tendo em vista que este foi criado para melhorar a precisão do diagnóstico de dor em crianças e promover suporte para a tomada de decisão no manejo da dor infantil (Silva, et al., 2020).

As tecnologias assistenciais são aquelas que incluem a construção de um saber técnico-científico resultante de investigações, aplicações de teorias e da experiência cotidiana dos profissionais. Para utilização de novas tecnologias assistenciais é necessário que seu conteúdo seja previamente validado. A validação do conteúdo se deu através da análise de especialistas. A seleção de profissionais experientes e de diferentes formações acadêmicas assegura maior acurácia ao processo, portanto, a multidisciplinaridade e a vasta experiência clínica dos avaliadores foram essenciais para validação do aplicativo, garantindo rigor metodológico necessário à validação (Niesthce, et al., 2015).

Para análise desta pesquisa, utilizamos o IVC. Quando analisado o IVC neste estudo, este obteve valor de IVC = 1.0 para todos os itens pesquisados, o que caracteriza o conteúdo do aplicativo como excelente. O IVC $=1.0$ indica que os avaliadores consideraram as informações contidas no aplicativo relevantes e que esta tecnologia pode ser usada como ferramenta na prática clínica (Hortense, et al., 2018).

A validação do conteúdo se deu por meio de doze questões semiestruturadas, divididas em categorias de funcionalidade, usabilidade e eficiência. Na validação, destaca-se na categoria de funcionalidade o item "facilidade no uso do aplicativo", na categoria usabilidade o item "facilidade em aprender a usar o aplicativo" e na categoria eficiência o item "tempo de execução adequado". A concordância total quanto a facilidade do uso e do aprendizado do aplicativo, minimiza o risco de limitações de uso, de aprendizado e de entendimento do conteúdo, facilitando a aceitação e dando credibilidade ao instrumento (Salome \& Alves, 2020)

O item "recursos adequados" da categoria eficiência, apesar de ter sido aprovado com IVC=1.0, foi o quesito que apresentou a menor taxa de concordância total. Os avaliadores relataram que a imagem utilizada na escala de mapa corporal poderia ser mais lúdica para a criança, de tamanho ampliado ou que a seleção do local de dor fosse realizada diretamente na figura.

Nossos resultados evidenciam que a linguagem, o conteúdo e o desenho do aplicativo foram transmitidos de forma clara e objetiva aos validadores, favorecendo a compreensão das informações técnicas. O reconhecimento das potencialidades e limitações do aplicativo durante o manuseio, demonstram que os profissionais estão aptos a inserção dessa ferramenta na rotina de trabalho (Ostrovsky, et al., 2016).

O aplicativo dispõe de quatro escalas unidimensionais para avaliação e quantificação da dor em crianças, sendo que três escalas avaliam intensidade da dor (EFWB, EVN e EVA) e uma ferramenta para localização da dor (Mapa corporal). As escalas são sempre mostradas na mesma ordem cronológica; segundo estudos prévios, a ordem de apresentação dos instrumentos não interfere no relato das respostas (Miró, et al., 2016).

Castarlenas, et al. (2015), construíram aplicativo para avaliação de dor em adolescentes com EVN e demonstraram forte correlação entre versão eletrônica e original com preferência dos entrevistados em usar o aplicativo. Wood, et al. (2011), ao utilizarem EFDR eletrônica e original com crianças, também encontraram resultados semelhantes de forte correlação, com preferência das crianças pelo uso da escala em versão eletrônica.

Estes resultados, corroboram com a iniciativa desta pesquisa, portanto, a substituição das escalas em papel pelo aplicativo eletrônico é aceitável. Os aplicativos tecnológicos possuem maior aceite das crianças e adolescentes, tem potencial de reduzir erros humanos na avaliação e podem melhorar a cooperação da criança (Tutelman, et al., 2018). 
Algumas escalas de avaliação de dor não compuseram o aplicativo. Dentre estas, temos a escala alógica de cores (EAC) a qual foi observado entre as escalas em papel e eletrônica, ainda que uma divergência pequena, porém significativa entre a pontuação da EAC eletrônica e a original, o que pode ter ocorrido pela condensação da escala original em formato eletrônico, pela redução do tamanho da escala ou por pequenos movimentos dos dedos das crianças produzirem grandes mudanças no resultado da dor (Sun, et al., 2015).

Dentre as escalas não incluídas no aplicativo, tem-se também a escala de fichas de pôquer (EFP). Apesar de sua facilidade de uso, atualmente é pouco utilizada na prática; revisão recente evidenciou que há poucas pesquisas abordando essa escala nos últimos anos, além disso, não há validação para uso em dores crônicas de crianças e não encontramos estudos de dispositivos eletrônicos com uso da EFP para comparação, provavelmente porque o uso da ficha real de pôquer seja importante para a interpretação da escala de avaliação (Zielinski, et al, 2020).

Dentre as escalas de faces validadas, as mais utilizadas em pesquisa clínica são as escalas EFDR e EFWB; devido à similaridade das escalas, tendo em vista que ambas são compostas por seis faces de "sem dor" até "dor intensa", optamos pelo uso de apenas uma, sendo esta a EFWB. Apesar de revisores expressarem preocupação com as faces de sorriso e choro poderem causar confusão entre intensidade da dor e afeto, nos testes com o aplicativo, foi a escala que apresentou melhor enquadramento na tela e facilidade de visualização e diferenciação no aplicativo (Manworren \& Stinson, 2017).

Em relação a equivalência e concordância das escalas de intensidade da dor, os resultados das pesquisas são controversos, a significância de variabilidade entre escalas adotadas nas pesquisas apresenta variações e não há consenso na literatura sobre o assunto. Apesar de similaridade entre as escalas de avaliação, elas não são substituíveis, portanto, criamos o aplicativo com diversidade das principais escalas de intensidade de dor, pois não há um instrumento que predomine ou substitua os demais, desse modo, o avaliador poderá dispor de um leque de opções para mensuração da dor (Myrvik, et al., 2015).

Não houve necessidade de diferenciação do aplicativo para gênero, pesquisas recentes não demonstram diferenciação na representação da intensidade ou qualidade da dor aguda entre meninos e meninas, sendo assim é desnecessário qualquer tipo de particularidade para gênero no aplicativo (Guedes, 2016).

Ao final do processo de avaliação, o aplicativo gera um relatório de dor com dados pessoais do paciente, data da avaliação e o resultado de cada escala. A medida em que, o profissional realizar avaliações subsequentes de cada paciente, terá um encadeamento de relatórios com acesso rápido em seu celular que lhe permitirá conhecer melhor a variabilidade da dor de cada paciente, analisar eficácia do tratamento e nortear a terapia analgésica com maior fundamentação de dados.

A grande maioria dos aplicativos móveis em saúde é voltado a equipe multiprofissional de saúde, fato este que corrobora a relevância desta pesquisa inovadora e inédita ao desenvolver uma ferramenta multiprofissional de avaliação de dor em crianças. Ainda nesse sentido, apesar do desenvolvimento de aplicativos para a equipe multiprofissional, há um predomínio de direcionamento para o público adulto, ainda que recentemente tenha aumentado o uso de aplicativos na área de pediatria. Nossa pesquisa não encontrou nenhum outro estudo brasileiro nessa vertente de criação de aplicativo multiprofissional no contexto da dor infantil, nesse sentido o aplicativo ganha ainda maior notoriedade, visto ter sido desenvolvido para uma abordagem bem específica e seguir as tendências globais de pesquisa de aplicativos de saúde em pediatria estando na vanguarda deste movimento a nível nacional e regional (Tibes, et al., 2014).

A presente pesquisa apresentou limitações quanto a seleção de profissionais avaliadores e aplicação dos testes. A seleção de avaliadores foi realizada por amostra de conveniência, o que pode ter interferido nos resultados obtidos. Não houve tempo hábil na pesquisa para validação do uso com o público alvo, as crianças, portanto, mais pesquisas devem ser feitas para avaliar a aplicabilidade na rotina clínica deste aplicativo. 


\section{Conclusão}

O aplicativo foi desenvolvido com rigor metodológico e pautado em um referencial teórico seguro, trata-se de uma inovação na prática pediátrica de avaliação da dor em crianças e que se mostrou como material válido segundo o índice de validade de conteúdo (IVC), apresentando IVC de 1.0 na avaliação dos especialistas multiprofissionais de saúde.

Acredita-se que o uso deste material poderá contribuir para a melhoria do manejo da dor pediátrica, no sentido de facilitar, padronizar e dinamizar o processo de avaliação por meio de escalas em formato eletrônico e promover maior envolvimento da criança no processo de cuidado da dor. Espera-se que o aplicativo móvel seja um grande estímulo para uma maior produção de trabalhos nessa área temática, podendo-se assim, aproveitar a imersão das tecnologias em saúde para promover o uso de ferramentas voltadas ao cuidado da dor em crianças.

\section{Referências}

Castarlenas, E., Sanchez-rodriguez, E., Vega, R., Roset, R., \& Miró, J. (2015). Agreement between verbal and electronic versions of the Numerical Rating Scale (NRS-11) when used to assess pain intensity in adolescents. Clinical Journal Pain, 31(3), 229-234. 10.1097/AJP.0000000000000104

Hortense, F. L. P., Bergerot, C. D., \& Domenico, E. B. L. (2018). Construction and validation of clinical contents for development of learning objects. Revista Brasileira de Enfermagem, 71(2), 306-313. doi.org/10.1590/0034-7167-2016-0622

Fortier, M. A., Chung, W. W., Martinez, A., Gago-masague, S., \& Sender, L. (2016). Pain Buddy: A novel use of m-health in the management of children's cancer pain. Computers in Biology and Medicine, 76(1), 202-214. 10.1016/j.compbiomed.2016.07.012

Guedes, D. M. B. (2016) Avaliação da dor de crianças: validação semântica dos cartões de qualidade da dor. Dissertação (Mestrado em Ciências) - Escola de Enfermagem da Universidade de São Paulo. São Paulo.

IPDMAQ - Instituto de Pesquisa e Desenvolvimento Tecnológico da Indústria de Maquinas e Equipamentos. O que é inovação? Acesso em 24/02/2020 de: http://www.ipdmaq.org.br/site.aspx/0-quee-inovacao.

Manworren, R. C. B., \& Stinson, J. (2016). Seminars in Pediatric Neurology Pediatric Pain Measurement, Assessment and Evaluation. Seminars in Pediatric Neurology, 23(3), 189-200. 10.1016/j.spen.2016.10.001

Marec-bernard, P., Gomez, F., Combet, S., Thibault, P., Moine, P. L., \& Bergeron, C. (2015). HEDEN Pain scale: A shortened behavioral scale for assessment of prolonged cancer or postsurgical pain in children aged 2 to 6 years. Pediatric Hematology and Oncology, 32(5), 291-303. 10.3109/08880018.2015.1005324

Miró, J., Castarlenas, E., De La Vega, R., Solé, E., Tomé-pires, C., Jensen, M. P., Engel, J. M., \& Racine, M. (2016) Validity of three rating scales for measuring pain intensity in youths with physical disabilities. European Journal of Pain, 20(1), 130-137. 10.1002/ejp.704

Myrvik, M. P., Drendel, A. L., Brandow, A. M., Yan, K., Hoffmann, R. G., \& Panepinto, J. A. (2015). A comparison of pain assessment measures in pediatric sickle cell disease: Visual Analog Scale versus Numeric Rating Scale. Journal of Pediatric Hematology and Oncology, 37(3), 190-194. 10.1097/mph.0000000000000306

Nietsche, E. A., Backes, V. M. S., Colomé, C. L. M., Ceratti R. N., \& Ferraz, F. (2005). Tecnologias educacionais, assistenciais e gerenciais: Uma reflexão a partir da concepção dos docentes de enfermagem. Revista Latino-americana de Enfermagem, 13(3), 344-352. doi.org/10.1590/S010411692005000300009

Ostrovsky, Y., Buttaro, T. M., Diamond, J., \& Hayes, J. (2016). Technology and dynamic pathways: how to improve nursing care, documentation, and efficiency. Iproc, 2(1), e. 31. 10.2196/iproc.6158

Pasquali, L. (2010). Instrumentação psicológica: fundamentos e práticas. Artmed.

Sallum, A. M. C., Garcia, D. M., \& Sanches, M. (2012). Dor aguda e crônica: Revisão narrativa da literatura. Acta Paulista de Enfermagem, 25(1), 150-154. doi.org/10.1590/S0103-21002012000800023

Salome, G. M., \& ALVES, N. F. (2020). App "SICKSEG" in mobile platforms for the prevention of skin injuries. Journal of Nursing UFPE on line, 14, e.244152. doi.org/10.5205/1981-8963.2020.244152

Santos, A. C. A., Melo, G. C. S., Inácio, M. S., Nascimento, G. C., Menezes, M. G. V., Santos, A. F., \& Machado, R. R. (2017). Conhecimento dos profissionais de enfermagem sobre as escalas de avaliação da dor em pediatria. Journal of Health Connections, 1(1), 19-32.

Sarno, F., Canella, D., \& Bandoni, D. H. (2014). Mobile health and excess weight: a systematic review. Revista Panamericana de Salud Publica, 35(5-6), 424431.

Silva, R. H., Gatti, M. A. N., Marta, S. N., Marafon, R. G. C., Neto, G. G. G., Andrade, E. B. O., Andrade, S. C., \& Lopes, V. C. N. (2020). Health applications for mobile devices: An integrative review. Brazilian Journal of Health Review, 3(5), 11754-1165. doi.org/10.34119/bjhrv3n5-033

Stinson, J. N., Jibb, L. A., Nguyen, C., Nathan, P. C., Maloney, A. M., Dupuis, L. L., Gerstle, J. T., Hopyan. S., Alman, B. A., Strahlendorf, C., Portwine, C., \& Johnston, D. L. (2015). Construct validity and reliability of a real-time multidimensional smartphone app to assess pain in children and adolescents with cancer. Pain. 156(12), 2607-2615. 10.1097/j.pain.0000000000000385 
Research, Society and Development, v. 10, n. 10, e529101017721, 2021

(CC BY 4.0) | ISSN 2525-3409 | DOI: http://dx.doi.org/10.33448/rsd-v10i10.17721

Sun, T., West, N., Ansermino, J. M., Montgomery, C. J., Myers, D., Dunsmuir, D., Lauder, G. R., \& Von Baeyer, C. L. (2015). A smartphone version of the Faces Pain Scale-Revised and the Color Analog Scale for postoperative pain assessment in children. Pediatric Anesthesia, 25(12), 1264-1273. $10.1111 /$ pan. 12790

Tibes, C. M. S. (2014). Aplicativo móvel para prevenção e classificação de úlceras de pressão. Dissertação (Mestrado em Ciências da Saúde). Universidade Federal de São Carlos. São Carlos.

Tibes, C. M. S, Dias, J. D, \& Zem-mascarenhas. S. H. (2014). Aplicativos móveis desenvolvidos para a área da saúde no Brasil: revisão integrativa da literatura. Revista Mineira de Enfermagem, 18(14), 471-478. doi.org/10.5935/1415-2762.20140035

Tutelman, P. R., Chambers, C. T., Stinson, J. N., Parker, J. A., Barwick, M., Witteman, H. O., Jibb, L., Stinson, H. C., Fernandez, C. V., Nathan, P. C., Campbell, F., \& Irwin, K. (2018). The implementation effectiveness of a freely available pediatric cancer pain assessment app: A pilot implementation study. JMIR Cancer, 4(2), e10280. 10.2196/10280

Wood, C., Von Baeyer C. L., Falinower, S., Moyse, D., Annequin, D., \& Legout, V. (2011). Electronic and paper versions of a faces pain intensity scale: concordance and preference in hospitalized children. BMC Pediatrics, 11(87), 1-9. 10.1186/1471-2431-11-87

Zielinski, J., Monika, M. K., \& Tomas, Z. (2020). Pain assessment and management in children in the postoperative period: A review of the most commonly used postoperative pain assessment tools, new diagnostic methods and the latest guidelines for postoperative pain therapy in children. Advances in clinical and experimental medicine, 29(3), 365-374. 10.17219/acem/112600 